\title{
Ophthalmic Examinations Group Identifier
}

National Cancer Institute

\section{Source}

National Cancer Institute. Ophthalmic Examinations Group Identifier. NCI Thesaurus.

Code C162012.

A character or string that represents an ophthalmic examinations group. 